Research Article

\title{
The Quantum Analysis of Nonlinear Optical Parametric Processes with Thermal Reservoirs
}

\author{
Tamirat Abebe $\mathbb{D}^{1},{ }^{1}$ Nebiyu Gemechu, ${ }^{1}$ Chimdessa Gashu $\left(\mathbb{D},{ }^{1}\right.$ Kebede Shogile, ${ }^{1}$ \\ Solomon Hailemariam, ${ }^{1}$ and Shimelis Adisu ${ }^{2}$ \\ ${ }^{1}$ Department of Physics, Jimma University, P.O. Box 378, Jimma, Ethiopia \\ ${ }^{2}$ Department of Chemistry, Jimma University, P.O. Box 378, Jimma, Ethiopia \\ Correspondence should be addressed to Tamirat Abebe; tam1704@gmail.com
}

Received 11 February 2020; Revised 22 April 2020; Accepted 4 May 2020; Published 14 May 2020

Academic Editor: Wonho Jhe

Copyright (c) 2020 Tamirat Abebe et al. This is an open access article distributed under the Creative Commons Attribution License, which permits unrestricted use, distribution, and reproduction in any medium, provided the original work is properly cited.

In this paper, employing the stochastic differential equations associated with the normal ordering, the quantum properties of a nondegenerate three-level cascade laser with a parametric amplifier and coupled to a two-mode thermal reservoir are thoroughly analyzed. Particularly, the enhancement of squeezing and the amplification of photon entanglement of the two-mode cavity light are investigated. It is found that the two cavity modes are strongly entangled and the degree of entanglement is directly related to the two-mode squeezing. Despite the fact that the entanglement and squeezing decrease with the increment of the mean photon number of the thermal reservoir, strong amount of these nonclassical properties can be generated for a considerable amount of thermal noise with the help of the nonlinear crystal introduced into the laser cavity. Moreover, the squeezing and entanglement of the cavity radiation enhance with the rate of atomic injection.

\section{Introduction}

Three-level cascade lasers have received considerable interest in connection with its potential as a source of light with interesting nonclassical features [1-6]. The quantum properties of the light is attributed to atomic coherence that can be induced either by preparing the atoms initially in a coherent superposition of the top and bottom levels [7] or coupling these levels by an external radiation [8] or using these mechanisms together [9].

Moreover, some authors have studied quantum properties of light generated by the three-level laser whose cavity contains a parametric amplifier [10-13]. A parametric amplifier involves three different modes of the radiation field, the signal, the idler, and the pump which are coupled by a nonlinear medium. In these devices, a pump photon interacts with a nonlinear crystal inside a cavity and is down converted into two highly correlated photons of different frequencies [12]. These works indicated the cavity radiation is found to be in squeezed and entangled states under certain conditions. In addition, the mean and variance of the photon number for a degenerate [11-13] and nondegenerate [14-19] three-level cascade laser whose cavity contains a parametric amplifier have been determined for different cases.

Furthermore, Tesfa [20] studied a two-photon correlated emission laser (CEL) in which the atomic coherence is initially prepared and the cavity is coupled to a two-mode thermal reservoir via a single-port mirror. He analyzed the effects of decoherence on entanglement in the two modes and evaluated the inseparability criterion for a continuous Gaussian state proposed earlier by Duan et al. [21]. He found that the generated light exhibits a two-mode squeezing and entanglement when initially there are more atoms at the lower level, even when the cavity is coupled to a thermal reservoir. Moreover, he also found that though the thermal noise entering the cavity degrades the squeezing and entanglement, it significantly increases the mean number of photon pairs of the superimposed radiation.

Moreover, Tesfa [22] studied the effect of the thermal light initially seeded in the cavity on the statistical and 
quantum features of the cavity radiation, but in the absence of the parametric amplifier. Thus, it has been shown that the degree of two-mode squeezing is almost independent of which mode is initially seeded, but the degree of entanglement decreases considerably when a light with the same strength is seeded in mode $b$. Moreover, the thermal light significantly damages two-mode squeezing and entanglement in the earlier stages of the lasing process. Thus, it is worthwhile to investigate the effect of thermal noise on these very sensitive nonclassical properties. On the other hand, the squeezing, entanglement, and statistical properties of the cavity radiation can be enhanced with the introduction of the parametric amplifier into the laser cavity. With this motivation, we are devoted to investigate the nonclassical properties of the light generated by a nondegenerate three-level laser whose cavity contains a nondegenerate parametric amplifier and coupled thermal reservoir.

In this study, the squeezing, entanglement, and statistical properties of the light produced by a nondegenerate three-level laser with a nondegenerate parametric amplifier and coupled to thermal reservoir are studied. We consider a nondegenerate three-level laser in which the pump mode emerging from the parametric amplifier does not couple the top and bottom levels of the injected atoms [17]. We carry out our analysis applying the pertinent master equation describing the dynamics of the optical device. The solutions for $c$-number cavity mode variables and correlation property of noise forces associated with normal ordering are determined. Using the resulting solutions and steady-state consideration, we obtained quadrature squeezing, the photon entanglement, the mean number of photon pairs, Mandel's Q-factor, and the second-order correlation functions of the two-cavity mode variables.

The paper is organized as follows. In the second section, the Hamiltonian and the model are presented, the master equation describing the dynamics of the optical device is derived, and the solutions of the cavity-mode variables are determined. The squeezing and entanglement of the twomode cavity radiation are analyzed in the third and fourth sections. In the fifth section, the statistical properties of the cavity radiation such as mean photon number, Mandel's $Q$-factor, and photon number correlation are studied.

\section{Hamiltonian and Master Equation}

We represent the top, intermediate, and bottom levels of a three-level atom in a cascade configuration by $|3\rangle,|2\rangle$, and $|1\rangle$, respectively, as shown in Figure 1. In addition, we assume the two modes $a_{1}$ and $a_{2}$ to be at resonance with the two transitions $|3\rangle \longrightarrow|2\rangle$ and $|2\rangle \longrightarrow|1\rangle$ dipole allowed, respectively, and direct transition between level $|3\rangle$ and level $|1\rangle$ to be dipole forbidden. The interaction of a nondegenerate three-level atom with the cavity modes can be described by the Hamiltonian in the interaction picture with the rotating and electric dipole approximation as

$$
\widehat{H}_{I}=i g\left[|3\rangle\left\langle 2\left|\widehat{a}_{1}-\widehat{a}_{1}^{\dagger}\right| 2\right\rangle\langle 3|+| 2\rangle\left\langle 1\left|\widehat{a}_{2}-\widehat{a}_{2}^{\dagger}\right| 1\right\rangle\langle 2|\right],
$$

where $g$ is a coupling constant, which is taken to be the same for both transitions, and $\widehat{a}_{1}$ and $\widehat{a}_{2}$ are the annihilation operators for the two cavity modes.
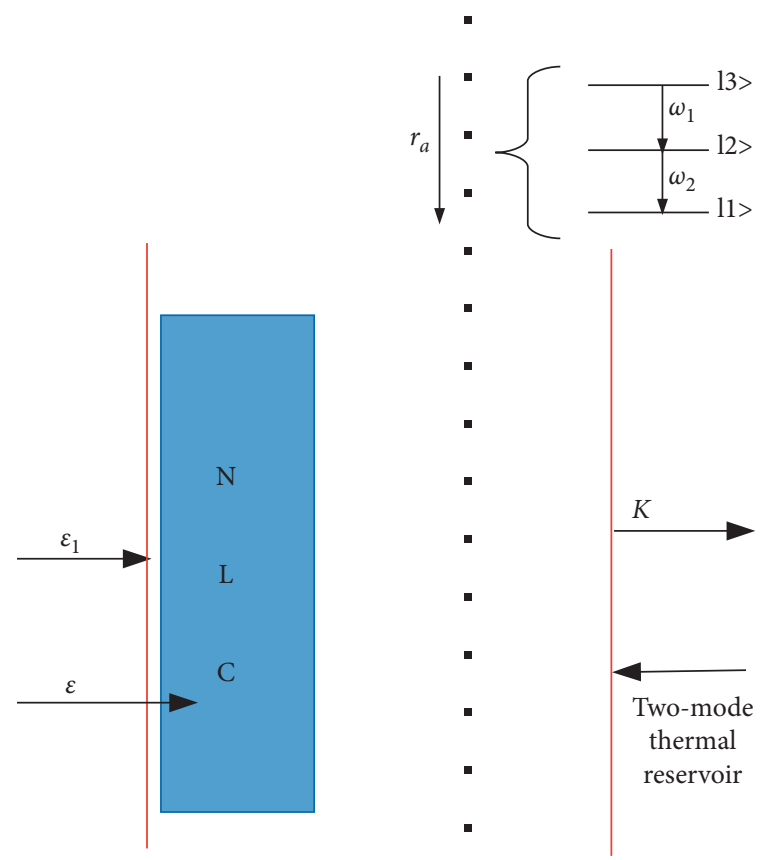

FIGURE 1: Schematic representation of a two-mode three-level cascade laser coupled with a two-mode thermal reservoir.

In this paper, we take the initial state of a three-level atom to be $\left|\psi_{A}(0)\right\rangle=C_{3}(0)|3\rangle+C_{1}(0)|1\rangle$, and hence, the initial density operator for a single atom has the form

$$
\widehat{\rho}_{A}(0)=\rho_{33}^{(0)}|3\rangle\left\langle 3\left|+\rho_{31}^{(0)}\right| 3\right\rangle\left\langle 1\left|+\rho_{13}^{(0)}\right| 1\right\rangle\left\langle 3\left|+\rho_{11}^{(0)}\right| 1\right\rangle\langle 1|,
$$

where $\rho_{33}^{(0)}=\left|C_{3}\right|^{2}$ and $\rho_{11}^{(0)}=\left|C_{1}\right|^{2}$ are, respectively, the probabilities for the atom to be initially in the upper and lower levels, and $\rho_{31}^{(0)}=C_{3} C_{1}^{*}$ and $\rho_{13}^{(0)}=C_{1} C_{3}^{*}$. Actually, this assumption corresponds to a situation in which the threelevel atom is initially prepared in a coherent superposition of the top and bottom levels. Experimental demonstration of coherent superpositions of the top and bottom levels of the atom was studied by Calderin et al. [23].

In addition, we seek to consider when such atoms are injected into a cavity at constant rate $r_{a}$ and removed after sometime $\tau$, which is long enough for the atoms to decay spontaneously to levels other than the middle or the lower level. The spontaneous decay rate $\gamma$ is taken to be the same for the two upper levels. In the good cavity limit, $\gamma \gg \kappa$, where $\kappa$ is the cavity damping constant, the cavity mode variables change slowly compared with the atomic variables. Hence, the atomic variables will reach steady state in relatively short time. The time derivative of such variables can then be set to zero, while keeping the remaining terms at time $t$. This procedure is referred to as the adiabatic approximation scheme. Since the coupling constant is taken to be small, we restrict ourselves to a linear analysis that amounts to dropping the higher order terms in $g$. Employing the linear and adiabatic approximation schemes in the good cavity limit that the equation of evolution of the density operator for the cavity modes has, in the absence of damping through the coupled mirror, the form [16] 


$$
\begin{aligned}
\dot{\hat{\rho}}_{1}(t)= & \frac{A \rho_{33}^{(0)}}{2}\left[2 \widehat{a}_{1}^{\dagger} \widehat{\rho} \widehat{a}_{1}-\widehat{a}_{1} \widehat{a}_{1}^{\dagger} \widehat{\rho}-\widehat{\rho} \widehat{a}_{1} \widehat{a}_{1}^{\dagger}\right] \\
& +\frac{A \rho_{11}^{(0)}}{2}\left[2 \widehat{a}_{2} \widehat{\rho} \widehat{a}_{2}^{\dagger}-\widehat{a}_{2}^{\dagger} \widehat{a}_{2} \widehat{\rho}-\widehat{\rho} \widehat{a}_{2}^{\dagger} \widehat{a}_{2}\right] \\
& -\frac{A \rho_{31}^{(0)}}{2}\left[2 \widehat{a}_{2} \widehat{\rho} \widehat{a}_{1}-\widehat{\rho} \widehat{a}_{1} \widehat{a}_{2}-\widehat{a}_{1} \widehat{a}_{2} \hat{\rho}\right] \\
& -\frac{A \rho_{13}^{(0)}}{2}\left[2 \widehat{a}_{1}^{\dagger} \widehat{\rho} \widehat{a}_{2}^{\dagger}-\widehat{\rho} \widehat{a}_{1}^{\dagger} \widehat{a}_{2}^{\dagger}-\widehat{a}_{1}^{\dagger} \widehat{a}_{2}^{\dagger} \hat{\rho}\right]
\end{aligned}
$$

where $A=\left(2 g^{2} r_{a} / \gamma^{2}\right)$ is the linear gain coefficient and for convenience we have set $\rho_{31}^{(0)}=\rho_{31}^{(0) *}$.

In order to study the dynamics of the cavity radiation of the combined system, it is necessary to obtain the corresponding equations of evolution. To begin with, the contribution of the initial thermal light in the cavity and the twomode vacuum reservoir to the master equation are sought. To this end, one can start with the well-established fact that the time evolution of the reduced density operator for the cavity radiation coupled to a reservoir has, in the Born approximation [16], the form

$$
\begin{aligned}
\dot{\hat{\rho}}_{2}(t)= & -i\left[\widehat{H}_{S}(t), \hat{\rho}(t)\right]-i\left[\left\langle\widehat{H}_{S R}(t)\right\rangle_{R}, \hat{\rho}(0)\right] \\
& -\int_{0}^{t}\left[\left\langle\widehat{\rho}_{S R}(t)\right\rangle_{R},\left[\widehat{H}_{S}\left(t^{\prime}\right), \hat{\rho}\left(t^{\prime}\right)\right]\right] \mathrm{d} t^{\prime} \\
& -\int_{0}^{t} \operatorname{Tr}_{R}\left[\widehat{H}_{S R}\left(t^{\prime}\right),\left[\widehat{H}_{S R}\left(t^{\prime}\right), \hat{\rho}\left(t^{\prime}\right) \widehat{R}\right]\right] \mathrm{d} t^{\prime},
\end{aligned}
$$

where $S$ and $R$ refer to the system and reservoir variables and $\hat{\rho}(0)$ represents the radiation initially in the cavity. Here, we consider the reservoirs to be composed of large number of submodes. Thus, the interaction of a two-mode cavity radiation with a two-mode thermal reservoir can be described in the interaction picture by a Hamiltonian of the form

$$
\begin{aligned}
\widehat{H}_{S R}= & i \sum_{j} \lambda_{j}\left[\hat{a}_{1}^{\dagger} \widehat{a}_{1 j} e^{i\left(\omega_{0}-\omega_{j}\right) t}-\widehat{a}_{1} \hat{a}_{1 j}^{\dagger} e^{-i\left(\omega_{0}-\omega_{j}\right) t}\right. \\
& \left.+\hat{a}_{2}^{\dagger} \widehat{a}_{2 j} e^{i\left(\omega_{0}-\omega_{j}\right) t}-\hat{a}_{2} \hat{a}_{2 j}^{\dagger} e^{-i\left(\omega_{0}-\omega_{j}\right) t}\right],
\end{aligned}
$$

where $\omega_{0}=\left(\omega_{1}+\omega_{2}\right) / 2$, with $\omega_{1}$ and $\omega_{2}$ representing the frequencies of the cavity radiations, $\left(\widehat{a}_{1 j}, \widehat{a}_{2 j}\right)$ are the annihilation operators of the two-mode thermal reservoir, $\omega_{j}$ is the frequency, and $\lambda_{j}$ is the coupling constant for the $j^{\text {th }}$ mode of the reservoir. With the aid of equation (5), one can write

$$
\begin{aligned}
\left\langle\widehat{H}_{S R}\right\rangle_{R}= & i \sum_{j} \lambda_{j}\left[\hat{a}_{1}^{\dagger}\left\langle\hat{a}_{1 j}\right\rangle_{R} e^{i\left(\omega_{0}-\omega_{j}\right) t}-\widehat{a}_{1}\left\langle\hat{a}_{1 j}^{\dagger}\right\rangle_{R} e^{-i\left(\omega_{0}-\omega_{j}\right) t}\right. \\
& \left.+\hat{a}_{2}^{\dagger}\left\langle\widehat{a}_{2 j}\right\rangle_{R} e^{i\left(\omega_{0}-\omega_{j}\right) t}-\widehat{a}_{2}\left\langle\hat{a}_{2 j}^{\dagger}\right\rangle_{R} e^{-i\left(\omega_{0}-\omega_{j}\right) t}\right] .
\end{aligned}
$$

Using the density operator of the thermal reservoir,

$$
\widehat{R}=\sum_{n=0}^{\infty} \frac{\bar{n}_{t h}^{n}}{\left(1+\bar{n}_{t h}\right)^{n+1}}|n\rangle\langle n|,
$$

one can easily obtain

$$
\begin{aligned}
\left\langle\hat{a}_{1 j}\right\rangle_{R} & =\left\langle\hat{a}_{2 j}\right\rangle_{R}=\left\langle\hat{a}_{1 j}^{2}\right\rangle_{R}=\left\langle\hat{a}_{2 j}^{2}\right\rangle_{R}=0, \\
\left\langle\hat{a}_{1 j}^{\dagger} \widehat{a}_{2 j}\right\rangle_{R} & =\left\langle\hat{a}_{2 j}^{\dagger} \widehat{a}_{1 j}\right\rangle_{R}=0, \\
\left\langle\hat{a}_{1 j} \widehat{a}_{2 j}\right\rangle_{R} & =\left\langle\hat{a}_{2 j}^{\dagger} \widehat{a}_{1 j}^{\dagger}\right\rangle_{R}=0, \\
\left\langle\hat{a}_{1 j}^{\dagger} \widehat{a}_{a 1 j}\right\rangle_{R} & =\left\langle\hat{a}_{2 j}^{\dagger} \widehat{a}_{a 2 j}\right\rangle_{R}=\bar{n}_{t h} .
\end{aligned}
$$

In addition, with the help of equation (11) along with the commutation relation $\left[\widehat{a}_{1 j}, \widehat{a}_{1 j}^{\dagger}\right]=\left[\widehat{a}_{2 j}, \widehat{a}_{2 j}^{\dagger}\right]=1$, one obtains $\left\langle\widehat{a}_{1 j} \hat{a}_{1 j}^{\dagger}\right\rangle=\left\langle\widehat{a}_{2 j} \hat{a}_{2 j}^{\dagger}\right\rangle=\bar{n}_{t h}+1$, where $\bar{n}_{t h 1}=\bar{n}_{t h 2}=\bar{n}_{t h}$ is the mean photon number of the thermal light. In view of these results, equation (6) reduces to

$$
\left\langle\widehat{H}_{S R}\right\rangle_{R}=0
$$

Therefore, the second commutation relation described in equation (4) is found to be zero. This confirms that the thermal light in the cavity does not directly contribute to the master equation. As a result, solving the remaining terms by following the standard approach yields

$$
\begin{aligned}
& \dot{\hat{\rho}}_{2}(t)=-i\left[\widehat{H}_{S}, \hat{\rho}(t)\right] \\
& +\frac{\kappa}{2} \bar{n}_{t h}\left[2 \widehat{a}_{1}^{\dagger} \widehat{\rho} \widehat{a}_{1}-\widehat{a}_{1} \widehat{a}_{1}^{\dagger} \widehat{\rho}-\widehat{\rho} \widehat{a}_{1} \widehat{a}_{1}^{\dagger}\right] \\
& +\frac{\kappa}{2} \bar{n}_{t h}\left[2 \widehat{a}_{2}^{\dagger} \widehat{\rho} \widehat{a}_{2}-\widehat{a}_{2} \widehat{a}_{2}^{\dagger} \widehat{\rho}-\widehat{\rho} \widehat{a}_{2} \widehat{a}_{2}^{\dagger}\right] \\
& +\frac{\kappa}{2}\left(\bar{n}_{t h}+1\right)\left[2 \widehat{a}_{1} \widehat{\rho} \widehat{a}_{1}^{\dagger}-\widehat{a}_{1}^{\dagger} \widehat{a}_{1} \hat{\rho}-\widehat{\rho} \widehat{a}_{1}^{\dagger} \widehat{a}_{1}\right] \\
& +\frac{\kappa}{2}\left(\bar{n}_{t h}+1\right)\left[2 \widehat{a}_{2} \widehat{\rho} \widehat{a}_{2}^{\dagger}-\widehat{a}_{2}^{\dagger} \widehat{a}_{2} \widehat{\rho}-\hat{\rho} \widehat{a}_{2}^{\dagger} \widehat{a}_{2}\right] .
\end{aligned}
$$

In addition, in the nondegenerate three-level laser a pump mode photon of frequency $\omega=\omega_{1}+\omega_{2}$ directly interacts with the nondegenerate parametric amplifier (NDPA) to produce the signal-idler photon pairs having the same frequencies as the two cavity modes [16, 17]. The interaction of the driving light modes, treated classically, with cavity modes, and the interaction of three-level atoms with a nondegenerate parametric amplifier can be described in the interaction picture by the quantum Hamiltonian.

$$
\widehat{H}_{S}=i \varepsilon_{1}\left[\widehat{a}_{1}^{\dagger}-\widehat{a}_{1}+\widehat{a}_{2}^{\dagger}-\widehat{a}_{2}\right]+i \frac{\varepsilon}{2}\left[\widehat{a}_{1}^{\dagger} \widehat{a}_{2}^{\dagger}-\widehat{a}_{1} \widehat{a}_{2}\right],
$$

where $\varepsilon_{1}$ is proportional to the amplitude of the driving light modes and $\varepsilon$ is considered to be real and constant and is proportional to the amplitude of the pump mode that drives the NLC (nonlinear crystal). Taking into account equations (3) and (13) along with (14), the master equation of the system turns out to be 


$$
\begin{aligned}
& \dot{\hat{\rho}}(t)=\varepsilon_{1}\left[\hat{\rho} \widehat{a}_{1}-\widehat{a}_{1} \hat{\rho}+\widehat{a}_{1}^{\dagger} \widehat{\rho}-\hat{\rho} \widehat{a}_{1}^{\dagger}+\widehat{\rho} \widehat{a}_{2}-\widehat{a}_{2} \widehat{\rho}+\widehat{a}_{2}^{\dagger} \widehat{\rho}-\widehat{\rho} \widehat{a}_{2}^{\dagger}\right] \\
& +\frac{\varepsilon}{2}\left[\hat{\rho} \widehat{a}_{1} \widehat{a}_{2}-\widehat{a}_{1} \hat{a}_{2} \hat{\rho}+\widehat{a}_{1}^{\dagger} \widehat{a}_{2}^{\dagger} \hat{\rho}-\hat{\rho} \hat{a}_{1}^{\dagger} \widehat{a}_{2}^{\dagger}\right] \\
& +\frac{\kappa}{2}\left(\bar{n}_{t h}+1\right)\left[2 \widehat{a}_{1} \widehat{\rho}_{1}^{\dagger}-\widehat{a}_{1}^{\dagger} \widehat{a}_{1} \widehat{\rho}-\widehat{\rho} \widehat{a}_{1}^{\dagger} \widehat{a}_{1}\right] \\
& +\frac{1}{2}\left(A \rho_{33}^{(0)}+\kappa \bar{n}_{t h}\right)\left[2 \widehat{a}_{1}^{\dagger} \hat{\rho} \widehat{a}_{1}-\widehat{a}_{1} \widehat{a}_{1}^{\dagger} \widehat{\rho}-\widehat{\rho} \widehat{a}_{1} \widehat{a}_{1}^{\dagger}\right] \\
& +\frac{1}{2}\left(A \rho_{11}^{(0)}+\kappa\left(\bar{n}_{t h}+1\right)\right)\left[2 \widehat{a}_{2} \hat{\rho} \widehat{a}_{2}^{\dagger}-\widehat{a}_{2}^{\dagger} \widehat{a}_{2} \hat{\rho}-\widehat{\rho} \hat{a}_{2}^{\dagger} \widehat{a}_{2}\right] \\
& +\frac{1}{2} \kappa \bar{n}_{t h}\left[2 \widehat{a}_{2}^{\dagger} \widehat{\rho}_{2}-\widehat{a}_{2} \widehat{a}_{2}^{\dagger} \widehat{\rho}-\widehat{\rho}_{2} \widehat{a}_{2}^{\dagger}\right] \\
& +\frac{A \rho_{31}^{(0)}}{2}\left[2 \widehat{a}_{2} \widehat{\rho}_{1}-\widehat{\rho} \widehat{a}_{1} \widehat{a}_{2}-\widehat{a}_{1} \widehat{a}_{2} \hat{\rho}\right] \\
& +\frac{A \rho_{13}^{(0)}}{2}\left[2 \widehat{a}_{1}^{\dagger} \widehat{\rho} \widehat{a}_{2}^{\dagger}-\widehat{\rho} \widehat{a}_{1}^{\dagger} \widehat{a}_{2}^{\dagger}-\widehat{a}_{1}^{\dagger} \hat{a}_{2}^{\dagger} \hat{\rho}\right] \text {. }
\end{aligned}
$$

Employing this master equation, the evolution of the two-mode cavity radiation in terms of $c$-number variables associated with the normal ordering $\alpha_{1}(t)$ and $\alpha_{2}(t)$ can be expressed in the form

$$
\begin{aligned}
& \dot{\alpha}_{1}(t)=-\gamma_{a} \alpha_{1}(t)+\nu_{-} \alpha_{2}^{*}(t)+\varepsilon_{1}+f_{1}(t), \\
& \dot{\alpha}_{2}^{*}(t)=-\gamma_{c} \alpha_{2}^{*}(t)+\nu_{+} \alpha_{1}(t)+\varepsilon_{1}+f_{2}^{*}(t),
\end{aligned}
$$

where $\gamma_{a}=(1 / 2)\left[\kappa-A \rho_{11}^{(0)}\right], \quad \gamma_{c}=(1 / 2)\left[\kappa+A \rho_{33}^{(0)}\right], \quad \nu_{ \pm}=$ $\varepsilon \pm(1 / 2) A \rho_{13}^{(0)}$, and $f_{1}(t)$ and $f_{2}^{*}(t)$ are the pertinent noise forces, the properties of which remain to be determined.

Following the straightforward procedure outlined in $[22,24]$, it is possible to obtain

$$
\begin{aligned}
& \alpha_{1}(t)=\Gamma_{+}(t) \alpha_{1}(0)+\chi_{+}(t) \alpha_{2}^{*}(0)+G_{1}(t)+\zeta_{+}, \\
& \alpha_{2}(t)=\Gamma_{-}(t) \alpha_{2}(0)+\chi_{-}(t) \alpha_{1}^{*}(0)+G_{2}(t)+\zeta_{-},
\end{aligned}
$$

in which

$$
\begin{aligned}
G_{1}(t)= & \int_{0}^{t}\left[\Gamma_{+}(t-t \prime) f_{1}(t \prime)+\chi_{+}\left(t-t^{\prime}\right) f_{2}^{*}(t \prime)\right] \mathrm{d} t \prime, \\
G_{2}(t)= & \int_{0}^{t}\left[\Gamma_{-}(t-t \prime) f_{2}^{*}(t \prime)+\chi_{-}(t-t \prime) f_{1}(t \prime)\right] \mathrm{d} t \prime, \\
\Gamma_{ \pm}(t)= & \frac{A_{ \pm}}{2 \lambda} e^{-(1 / 2) \lambda_{\mp} t}-\frac{A_{\mp}}{2 \lambda} e^{-(1 / 2) \lambda_{ \pm} t}, \\
\chi_{ \pm}(t)= & \frac{2 v_{\mp}}{2 \lambda} e^{-(1 / 2) \lambda_{-} t}-\frac{2 v_{\mp}}{2 \lambda} e^{-(1 / 2) \lambda_{+} t}, \\
\zeta_{ \pm}= & \frac{\varepsilon_{1}}{\lambda}\left[\frac{A_{+} \pm 2 \nu_{\mp}}{\lambda_{\mp}}\left(1-e^{-(1 / 2) \lambda_{\mp} \tau}\right)\right. \\
& \left.-\frac{A_{-} \pm 2 v_{\mp}}{\lambda_{ \pm}}\left(1-e^{-(1 / 2) \lambda_{ \pm} \tau}\right)\right], \\
\lambda_{ \pm}= & \frac{1}{2}(2 \kappa+A \eta \pm \lambda), \\
A_{ \pm}= & A \pm \sqrt{A^{2} \eta^{2}+16 \varepsilon^{2}},
\end{aligned}
$$

where the noise forces satisfy the following correlations:

$$
\begin{aligned}
\left\langle f_{1}(t)\right\rangle & =\left\langle f_{2}(t)\right\rangle=0, \\
\left\langle f_{1}\left(t^{\prime}\right) f_{1}(t)\right\rangle & =\left\langle f_{2}\left(t^{\prime}\right) f_{2}(t)\right\rangle=0, \\
\left\langle f_{1}^{*}(t) f_{2}\left(t^{\prime}\right)\right\rangle & =\left\langle f_{2}^{*}\left(t^{\prime}\right) f_{1}(t)\right\rangle=0, \\
\left\langle f_{1}^{*}(t) f_{1}\left(t^{\prime}\right)\right\rangle & =\left(A \rho_{11}^{(0)}+\kappa \bar{n}_{t h}\right) \delta\left(t-t^{\prime}\right), \\
\left\langle f_{2}^{*}\left(t^{\prime}\right) f_{2}(t)\right\rangle & =\kappa \bar{n}_{t h} \delta\left(t-t^{\prime}\right), \\
\left\langle f_{1}\left(t^{\prime}\right) f_{2}(t)\right\rangle & =\frac{1}{2}\left(2 \varepsilon+A \rho_{13}^{(0)}\right) \delta\left(t-t^{\prime}\right) .
\end{aligned}
$$

It proves to be useful to introduce a new parameter which relates the probabilities of the atom to be in the upper and lower levels. We define the parameter $\eta$ such that $\rho_{11}^{(0)}=$ $(1-\eta / 2)$ with $-1<\eta<1$. For three-level atoms initially in a coherent superposition of the top and bottom levels, one obtains $\rho_{33}^{(0)}=(1+\eta / 2)$, and in view of the relation $\left|\rho_{13}^{(0)}\right|^{2}=\rho_{11}^{(0)} \rho_{33}^{(0)}$, one easily finds $\rho_{13}^{(0)}=(1 / 2) \sqrt{1-\eta^{2}}$.

\section{Quadrature Variance}

Here, we seek to analyze the quadrature squeezing properties of the two-mode light in the cavity. The squeezing properties of the two-mode light in the cavity can be described by two quadrature operators defined as

$$
\begin{aligned}
& \widehat{c}_{+}=\widehat{c}^{\dagger}+\widehat{c}, \\
& \widehat{c}_{-}=i\left(\widehat{c}^{\dagger}-\widehat{c}\right),
\end{aligned}
$$

where $\widehat{c}=(1 / \sqrt{2})\left(\widehat{a}_{1}+\widehat{a}_{2}\right)$ is the annihilation operator for the two-mode cavity radiation. These quadrature operators satisfy the commutation relation $\left[\widehat{c}_{+}, \widehat{c}_{-}\right]=2 i$. On the basis of these definitions, a two-mode light is said to be in a two-mode squeezed state if either $\Delta c_{+}^{2}<1$ or $\Delta c_{-}^{2}<1$ $[19,24]$. The variances of the quadrature operators can be expressed as

$$
\Delta c_{ \pm}^{2}=\left\langle\widehat{c}_{ \pm}^{2}\right\rangle-\left\langle\widehat{c}_{ \pm}\right\rangle^{2} .
$$

It is possible to express the variance of the quadrature operators (21) and (22) in terms of the c-number variables associated with the normal ordering taking the cavity modes to be initially in a two-mode vacuum state, as

$$
\begin{aligned}
\Delta c_{ \pm}^{2}= & 1+\left\langle\alpha_{1}^{*}(t) \alpha_{1}(t)\right\rangle+\left\langle\alpha_{2}^{*}(t) \alpha_{2}(t)\right\rangle+2\left\langle\alpha_{1}(t) \alpha_{2}(t)\right\rangle \\
& \mp \frac{1}{2}\left[\left(\left\langle\alpha_{1}^{*}(t)\right\rangle+\left\langle\alpha_{2}^{*}(t)\right\rangle\right) \pm\left(\left\langle\alpha_{1}(t)\right\rangle+\left\langle\alpha_{2}(t)\right\rangle\right)\right] \\
& \pm\left[\left\langle\alpha_{1}^{2}(t)\right\rangle+\left\langle\alpha_{2}^{2}(t)\right\rangle+2\left\langle\alpha_{1}(t) \alpha_{2}(t)\right\rangle\right] .
\end{aligned}
$$

In view of equations (17) and (18), the steady-state quadrature variances are found to be 


$$
\begin{aligned}
\Delta c_{ \pm}^{2}= & 1+\frac{\kappa A(1-\eta)(4 \kappa+3 A \eta+A)+16 \varepsilon^{2}(\kappa+A \eta)}{4\left[\kappa(\kappa+A \eta)-4 \varepsilon^{2}\right](2 \kappa+A \eta)} \\
& +\frac{\kappa\left(4 \varepsilon+A \sqrt{1-\eta^{2}}\right)\left[4 \kappa^{2}(2 \kappa+2 A \eta)+A^{2}\right] 2 \kappa \bar{n}_{t h}}{4\left[\kappa(\kappa+A \eta)-4 \varepsilon^{2}\right](2 \kappa+A \eta)} \\
& \pm\left[\frac{\kappa\left(4 \varepsilon+A \sqrt{1-\eta^{2}}\right)(2 \kappa+A \eta+A)}{4\left[\kappa(\kappa+A \eta)-4 \varepsilon^{2}\right](2 \kappa+A \eta)}\right. \\
& \left.+\frac{\left[4 \varepsilon(2 \kappa+A \eta)+A^{2} \sqrt{1-\eta^{2}}\right] 2 \kappa \bar{n}_{t h}}{4\left[\kappa(\kappa+A \eta)-4 \varepsilon^{2}\right](2 \kappa+A \eta)}\right] .
\end{aligned}
$$

We clearly see from equation (25) that the quadrature variances are independent of the parameter $\varepsilon_{1}$ which represents the cavity driving coherent light. This shows that the cavity driving coherent light does not have any effect on the degree of squeezing of the two-mode light. This is due to the fact that the external driving coherent light does not introduce additional coherence to the system which is believed to be the source of squeezing in three-level cascade lasers [25].

As it can readily be seen from Figure 2, the two-mode cavity radiation produced by a nondegenerate three-level laser with a nondegenerate parametric amplifier and coupled to thermal reservoir exhibits squeezing for some values of the injected atomic coherence. One can observe that the degree of squeezing is significantly degraded by the thermal noise. For this case, the maximum quadrature squeezing is found to be nearly $70 \%$ when the cavity is coupled to a thermal reservoir with $\bar{n}_{t h}=0$ for $A=100, \kappa=0.8$, and $\varepsilon=$ 0.399 and occurs at $\eta=0.1$. Moreover, from the same figure, we observe that when the $\bar{n}_{t h}$ increases the degree of squeezing decreases. This is because of the effect of the thermal fluctuations arising from the heating due to vibration of the atoms on the walls of the cavity.

In Figure 3, we plot the variances of the minus quadrature $\Delta c_{-}^{2}$ versus $\eta$, the parameter for $A=100, \kappa=0.8, \bar{n}_{t h}=0.15$, and with different values of $\varepsilon$. The figure clearly shows that the effect of the parametric oscillator $\varepsilon$ is to increase the degree of squeezing for small values of $\eta$ as previously determined [17].

We clearly see from Figure 4 that the degree of squeezing increases with the linear gain coefficient and a substantial degree of squeezing is found for smaller values of $\eta$. This indicates that the more the atoms are injected into the cavity at a time the more the degree of the squeezing of the cavity radiation would be. The maximum squeezing occurs when the atoms are prepared with initial coherence very close to the maximum possible value in this case. In particular, a maximum of $72 \%$ squeezing occurs at $\eta=0.1$ for $A=1000, \kappa=0.8, \varepsilon=0.399$, and $\bar{n}_{t h}=0.15$.

We also plotted, in Figure 5, the variance of the minus squeezed quadrature versus the parameters $\varepsilon$ and $\eta$ for $A=100, \kappa=0.8$, and $\bar{n}_{t h}=0.15$. It is possible to see from this plot that the two-mode squeezing increases with the amplitude of the parametric oscillator $\varepsilon$ and for small values of $\eta$. Moreover, the value of $\eta$ at which the maximum squeezing occurs decreases to zero as $\varepsilon$ increases. Furthermore, Figure 5 clearly shows that the degree of squeezing increases due to the presence of the parametric oscillator.

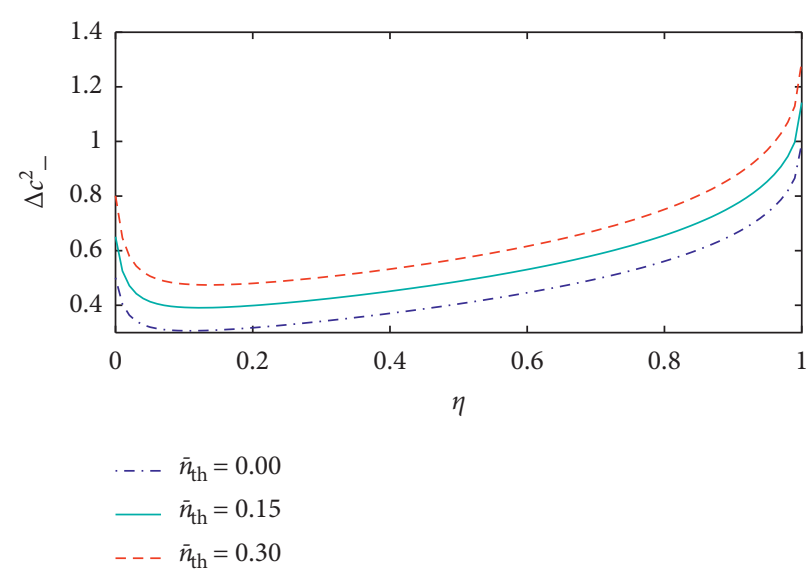

Figure 2: Plots of the quadrature variance (equation (25)) versus $\eta$ for $A=100, \kappa=0.8, \varepsilon=0.399$ and for different values of $\bar{n}_{t h}$.

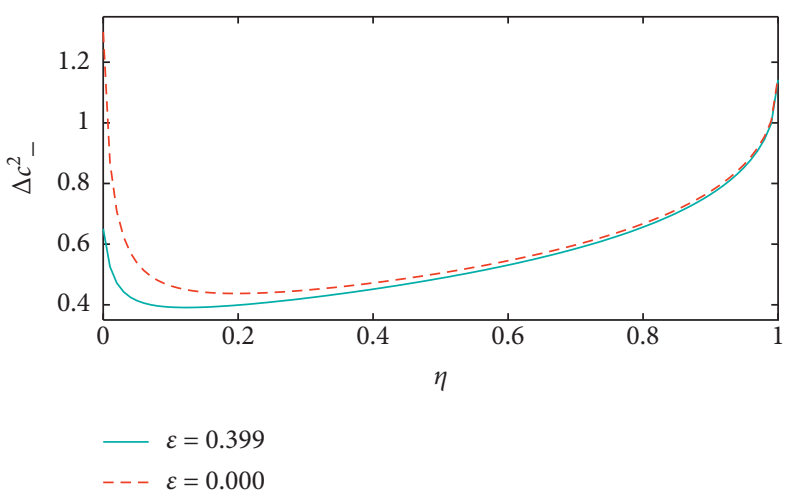

FIGURE 3: Plots of the minus quadrature variance (equation (25)) versus $\eta$ for $A=100, \kappa=0.8, \bar{n}_{t h}=0.15$ and for different values of $\varepsilon$.

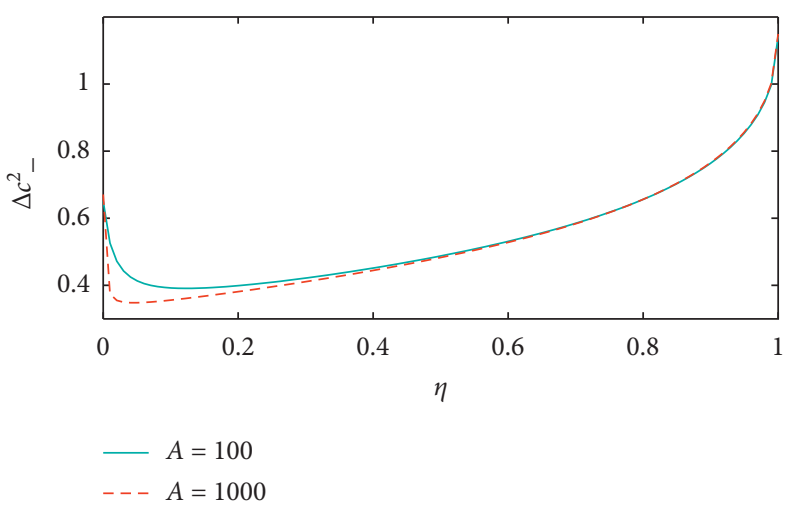

Figure 4: Plots of the minus quadrature variance (equation (25)) versus $\eta$ for $\kappa=0.8, \varepsilon=0.399, \bar{n}_{t h}=0.15$ and for different values of the linear gain coefficient.

\section{Detection of Entanglement}

For continuous variables photon entanglement, several sufficient inseparability criteria for a composite state have been proposed [21, 26-36]. The most relevant method is the logarithmic negativity which depicts the presence of 


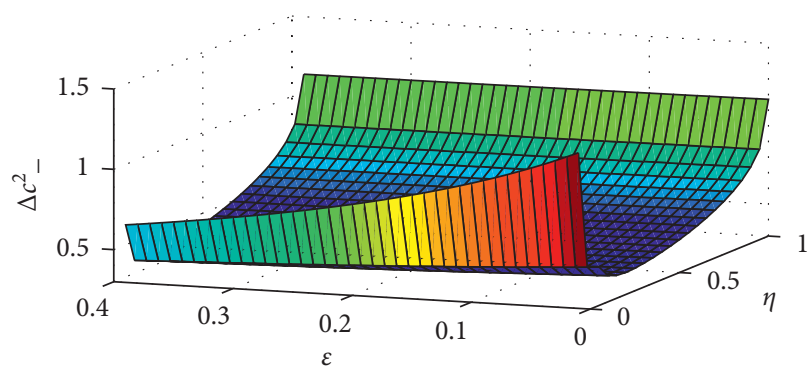

FIGURE 5: A plot of the minus quadrature variance (equation (25)) versus $\varepsilon$ and $\eta$ for $A=100, \kappa=0.8$, and $\bar{n}_{t h}=0.15$.

entanglement for two-mode continuous variables based on the negativity of the partial transposition $[22,37,38]$. The negative partial transpose must be parallel with respect to entanglement monotone in order to obtain the degree of entanglement. The logarithmic negativity is combined with negative partial transpose in another case where $V_{S}$ represents the smallest eigenvalue of the simplistic matrix [22]:

$$
V_{S}=\sqrt{\frac{\sigma-\sqrt{\left(\sigma^{2}-4 \operatorname{det} \Gamma\right)}}{2}},
$$

where the invariant and covariance matrices are, respectively, denoted as

$$
\begin{aligned}
& \sigma=\operatorname{det} \Sigma_{1}+\operatorname{det} \Sigma_{2}-2 \operatorname{det} \Sigma_{12}, \\
& \Gamma=\left(\begin{array}{cc}
\Sigma_{1} & \Sigma_{12} \\
\Sigma_{12}^{T} & \Sigma_{2}
\end{array}\right),
\end{aligned}
$$

in which $\Sigma_{1}$ and $\Sigma_{2}$ are the covariance matrices describing each mode separately while $\Sigma_{12}$ are the intermodal correlations. The elements of the matrix in equation (28) are given by

$$
\Gamma_{i j}=\frac{1}{2}\left\langle\widehat{X}_{i} \widehat{X}_{j}+\widehat{X}_{j} \widehat{X}_{i}\right\rangle-\left\langle\widehat{X}_{i}\right\rangle\left\langle\widehat{X}_{j}\right\rangle
$$

in which $i, j=1,2,3,4$. The quadrature operators are defined as $\widehat{X}_{1}=\widehat{a}_{1}+\widehat{a}_{1}^{\dagger}, \widehat{X}_{2}=i\left(\widehat{a}_{1}^{\dagger}-\widehat{a}_{1}\right), \widehat{X}_{3}=\widehat{a}_{2}+\widehat{a}_{2}^{\dagger}$, and $\widehat{X}_{4}=i\left(\widehat{a}_{2}^{\dagger}-\widehat{a}_{2}\right)$. With this introduction, the extended covariance matrix, which can be expressed in terms of $c$-number variables associated with the normal ordering and nothing that $\left\langle\alpha_{1} \alpha_{2}\right\rangle=\left\langle\alpha_{1}^{*} \alpha_{2}^{*}\right\rangle$, goes over into

$$
\Gamma=\left(\begin{array}{cccc}
\Lambda & 0 & \chi & 0 \\
0 & \Lambda & 0 & -\chi \\
\chi & 0 & \Delta & 0 \\
0 & -\chi & 0 & \Delta
\end{array}\right),
$$

where $\Lambda=2\left\langle\alpha_{1}^{*} \alpha_{1}\right\rangle+1, \chi=2\left\langle\alpha_{1} \alpha_{2}\right\rangle, \Delta=2\left\langle\alpha_{2}^{*} \alpha_{2}\right\rangle+1$ are $c$-number variables associated with the normal ordering. The logarithmic negativity for a two-mode state is defined as

$$
E_{N}=\max \left[0,-\log _{2} V_{S}\right] \text {. }
$$

The entanglement is achieved when $E_{N}$ is positive within the region of the lowest eigenvalue of covariance matrix $V_{S}<1$.
Next on account of equation (29) along with the definitions of (31), one can readily show that

$$
\begin{aligned}
\operatorname{det} \Sigma_{1} & =\left[2\left\langle\alpha_{1}^{*} \alpha_{1}\right\rangle+1\right]^{2}, \\
\operatorname{det} \Sigma_{2} & =\left[2\left\langle\alpha_{2}^{*} \alpha_{2}\right\rangle+1\right]^{2}, \\
\operatorname{det} \Sigma_{12} & =-4\left\langle\alpha_{1} \alpha_{2}\right\rangle^{2} .
\end{aligned}
$$

It is also possible to establish that

$$
\operatorname{det} \Gamma=\left[\sqrt{\operatorname{det} \Sigma_{1} \operatorname{det} \Sigma_{2}}-\sqrt{\operatorname{det} \Sigma_{12}^{T} \operatorname{det} \Sigma_{12}}\right]^{2} \text {. }
$$

As it can be noted from Figure 6, the degree of entanglement increases for smaller values of the initial preparation of atoms but decreases for larger values. It can also be seen that larger values of the linear gain coefficient produces a robust entangled light. The maximum achievable degree of entangled light in this case is $96 \%$, and it occurs for $A=1000$ and $\eta=0.02$. This criterion also predicts the absence of entanglement for $\eta=1$ no matter how we manipulate the rate of atomic injection in the absence of a parametric amplifier.

It is not difficult to see from Figure 7 that the parametric amplifier produces a considerable degree of entangled light for very small value of the linear gain coefficient regardless of how atoms are initially prepared. The maximum achievable degree of entangled light in this case is $84.5 \%$, and it occurs for $\bar{n}_{t h}=0, A=100$, and $\eta=0.08$. Moreover, it can be seen in this figure that when the values of the mean photon number of the thermal reservoir increases, the degree of entanglement decreases.

On the other hand, Figure 8 shows that the effect of the parametric amplifier for a large value of the linear gain coefficient does not produce considerable changes to the degree of entanglement. Hence, the maximum achievable degree of entangled light in this case is $82 \%$, and it occurs for $A=100$ and $\eta=0.13$. The behaviors of a nondegenerate three-level laser with a nondegenerate parametric amplifier and non-degenerate three-level laser without the parametric amplifier appear to be the same for a very large value of the linear gain coefficient (rate of atomic injection) with exception of the existence of the entanglement at the minimum atomic coherence which represents absence of photons in the cavity.

Furthermore, it is clearly shown in Figure 9 that for large values of the linear gain coefficient $A$ and the parametric amplifier $\varepsilon$, the enhancement of the maximum possible degree of entanglement is occurred. However, for smaller values of the linear gain coefficient and the pumping mode, a robust entangled light is produced.

\section{Photon Statistics}

In this section, we seek to study the statistical properties of the cavity radiation of a three-level cascade laser, such as the mean photon number, Mandel's Q-factor, and the normalized second-order correlation function for the system under consideration. 


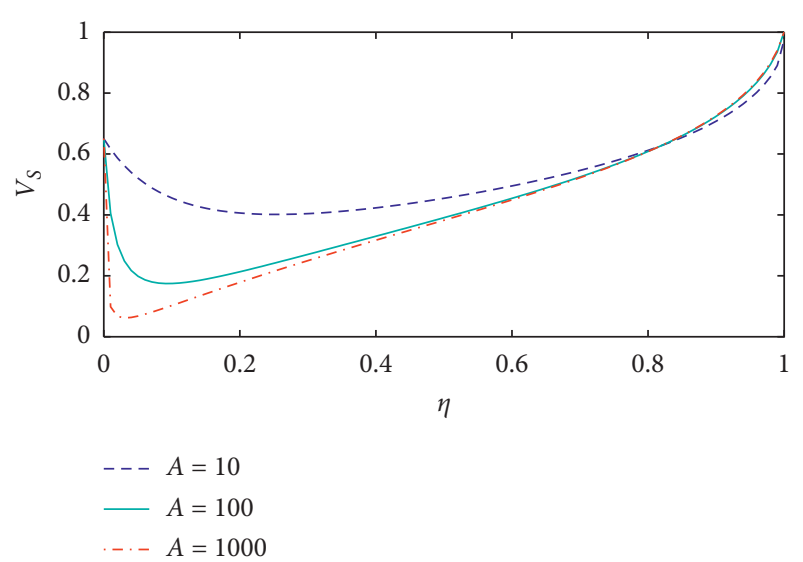

Figure 6: Plots of the smallest eigenvalue $V_{S}$ versus $\eta$ for $\bar{n}_{t h}=0.15$, $\kappa=0.8, \varepsilon=0.399$ and for different values of the linear gain coefficient.

5.1. Mean Photon Number. In order to know about the brightness of the generated light, it is necessary to study the mean number of photon pairs describing the two-mode cavity radiation that can be defined as

$$
\bar{N}=\left\langle\widehat{c}^{\dagger}(t) \widehat{c}(t)\right\rangle \text {. }
$$

It then follows that

$$
\bar{N}=\frac{1}{2}\left[\left\langle\alpha_{1}^{*}(t) \alpha_{1}(t)\right\rangle+\left\langle\alpha_{2}^{*}(t) \alpha_{2}(t)\right\rangle\right] .
$$

Since $\left\langle\alpha_{1}^{*}(t) \alpha_{1}(t)\right\rangle$ and $\left\langle\alpha_{2}^{*}(t) \alpha_{2}(t)\right\rangle$ represent the mean photon numbers in mode $a_{1}$ and mode $a_{2}$, respectively, $\bar{N}$ can be interpreted as the mean number of photon pairs. As it can be seen in equation (35), the term that contains $\varepsilon$ represents the contribution from the external driving coherent light of the parametric amplifier to the total mean photon number. Therefore, it is easy to verify that equation (35) represents the mean number of photon pairs of the system.

It is not difficult to see from Figure 10 that the mean number of photon pairs increases with the linear gain coefficient for $\kappa=0.8, \varepsilon=0.399, \bar{n}_{t h}=0.15$ and for smaller values of $\eta$, but it decreases for larger values. Moreover, Figure 11 clearly indicates that the mean photon number of the cavity modes increases with the amplitude of the coherent driving light $\varepsilon$. In order to clearly see by what extent the coherent fields enhance the mean photon number over the laser system, we plotted in Figure 12 the mean number of photon pairs versus $\varepsilon$ and $A$ for $\eta=0.1, \kappa=0.8$, and $\bar{n}_{t h}=0.15$. It is quite interesting to note from this figure that the coherent fields enhance the mean photon number over the laser system by several hundreds of mean photon numbers. Therefore, the increase in the mean photon number is observed in a region where the degree of twomode squeezing and entanglement is significant making the system under consideration a viable source of intense squeezed as well as entangled light. We hence note that coupling the cavity to the thermal reservoir increases the mean number of photon pairs, which we believe is an encouraging result.

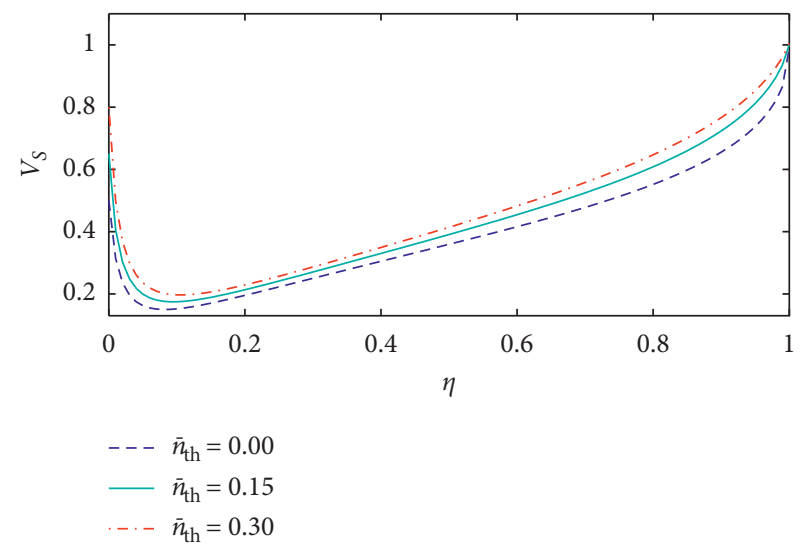

FIgURE 7: Plots of the smallest eigenvalue $V_{S}$ versus $\eta$ for $A=100$, $\kappa=0.8, \varepsilon=0.399$ and for different values of $\bar{n}_{t h}$.

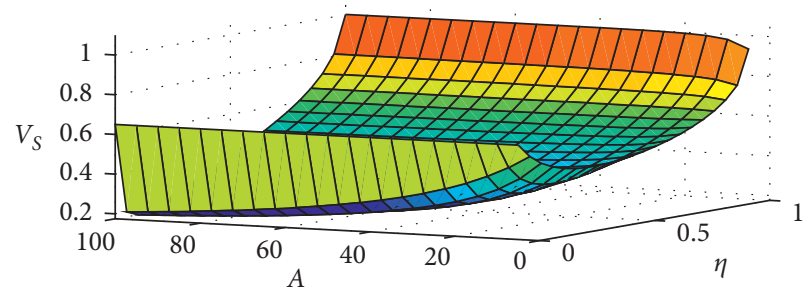

FIgURE 8: A plot of the smallest eigenvalue $V_{S}$ of the two-mode cavity radiation versus $A$ and $\eta$ for $\kappa=0.8, \varepsilon=0.399$, and $\bar{n}_{t h}=0.15$.

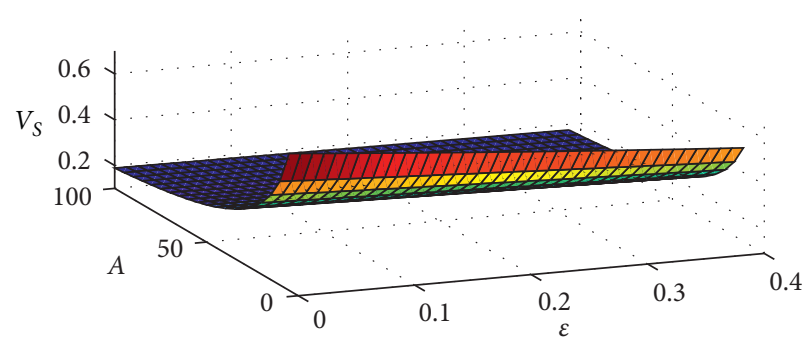

FIgURe 9: A plot of the smallest eigenvalue $V_{S}$ of the two-mode cavity radiation versus $A$ and $\varepsilon$ for $\kappa=0.8, \bar{n}_{t h}=0.15$, and $\eta=0.1$.

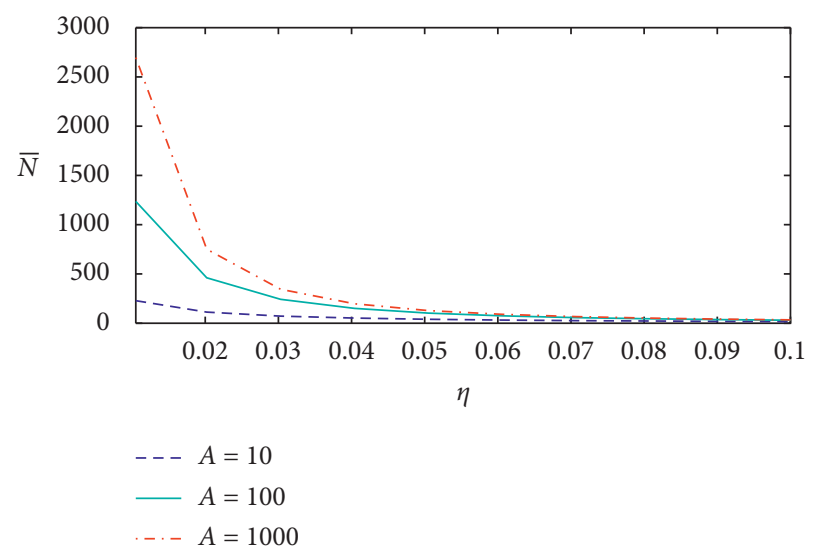

Figure 10: Plots of the mean number of photon pairs (equation (35)) versus $\eta$ for $\kappa=0.8, \varepsilon=0.399, \bar{n}_{t h}=0.15$ and for different values of linear gain coefficients. 


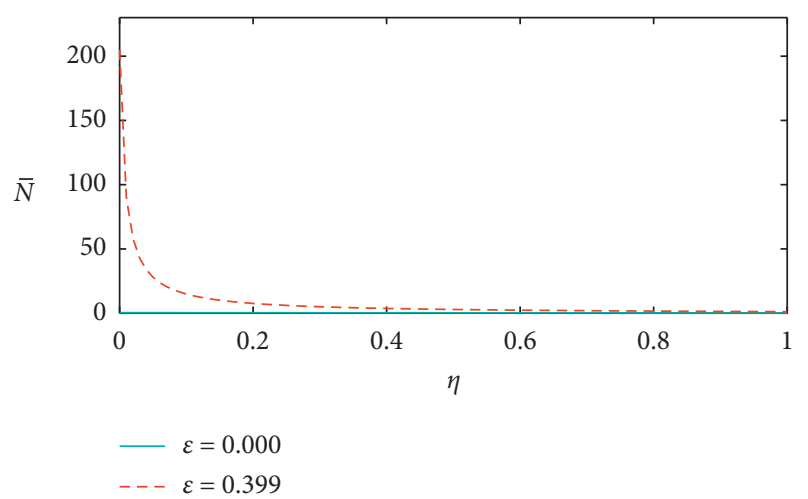

Figure 11: Plots of the mean number of photon pairs (equation (35)) versus $\eta$ for $A=10, \kappa=0.8, \bar{n}_{t h}=0.15$ and for different values of $\varepsilon$.

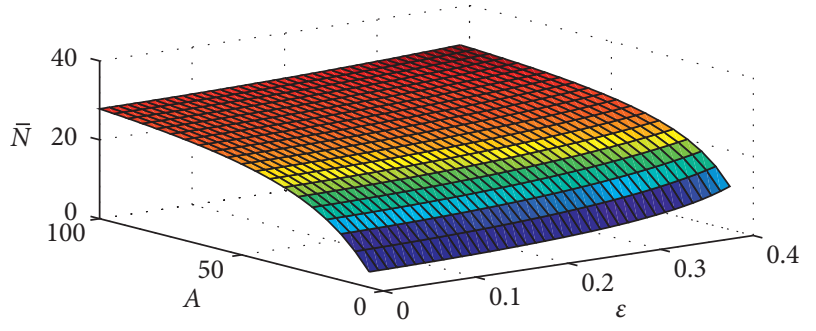

FIgURE 12: A plot of the mean number of photon pairs (equation (35)) versus $\varepsilon$ and $A$ for $\eta=0.1, \kappa=0.8$, and $\bar{n}_{t h}=0.15$.

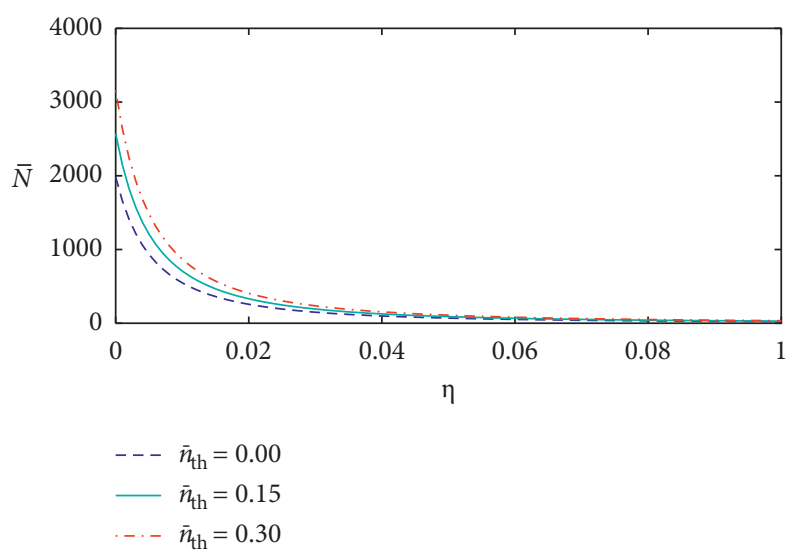

FIGURE 13: Plots of the mean number of photon pairs (equation (35)) versus $\eta$ for $A=100, \kappa=0.8, \varepsilon=0.0$ and for different values of $\bar{n}_{t h}=0.15$.

The result presented in Figure 13 indicates that the mean number of photon pairs increases with the mean photon number of the thermal reservoir at the minimum atomic coherence $\eta$. The maximum possible value of the mean number of photon pairs in this case occurs for $\bar{n}_{t h}=0.30$ and small $\eta=0$. This is readily understandable since traversing more thermal mean photon number $\bar{n}_{t h}$ across the cavity leads to the generation of more photons. We set $\varepsilon=0$ so that the dependence of the mean photon number on the parameter $\bar{n}_{t h}$ is clearly evident from the figure.
5.2. Mandel's Q-Factor. It is a common experience that a nonclassical photon number correlation can be studied applying the measure of the departure of the photon statistics from the Poisson character. This measure of departure can be represented by Mandel's Q-factor defined as

$$
Q=\frac{\left\langle(\Delta \widehat{n})^{2}\right\rangle-\langle\widehat{n}\rangle}{\langle\widehat{n}\rangle}
$$

where $\widehat{n}=\widehat{c}^{\dagger} \widehat{c}$ is the photon number operator of a two-mode cavity radiation. It is not difficult to verify that equation (36) can be expressed by putting the operators in the normal ordering as

$$
Q=\frac{\left\langle\widehat{c}^{\dagger 2} \widehat{c}^{2}\right\rangle+\left\langle\widehat{c}^{\dagger} \widehat{c}\right\rangle^{2}}{\left\langle\widehat{c}^{\dagger} \widehat{c}\right\rangle}
$$

where $\widehat{c}=(1 / \sqrt{2})\left(\widehat{a}_{1}+\widehat{a}_{2}\right)$ is the annihilation operator that describes the two-mode cavity radiation. With the help of this, the normal ordering of the operators would not be altered since $\widehat{a}_{1}$ and $\widehat{a}_{2}$ commute. Hence, it is possible to put the resulting expression in terms of $c$-number variables associated with the normal order as

$$
Q=\frac{\left\langle\gamma^{* 2}(t) \gamma^{2}(t)\right\rangle+\left\langle\gamma^{*}(t) \gamma(t)\right\rangle^{2}}{\left\langle\gamma^{*}(t) \gamma(t)\right\rangle}
$$

where $\gamma=(1 / \sqrt{2})\left(\alpha_{1}(t)+\alpha_{2}(t)\right)$. Hence, employing equations (17) and (18), one obtains

$$
Q=\bar{N}+\frac{\left\langle\alpha_{1}(t) \alpha_{2}(t)\right\rangle^{2}}{\bar{N}} .
$$

It is well known that the negativity of Mandel's parameter refers to a sub-Poissonian character of the photon statistics that essentially refers to a nonclassical property. Since the mean number of photon pairs and $\left\langle\alpha_{1}(t) \alpha_{2}(t)\right\rangle^{2}$ are positive, Mandel's $Q$-factor in this case is definitely greater than 0 . This ensures that the generated radiation demonstrates super-Poissonian photon statistics while exhibiting nonclassical properties such as squeezing and entanglement.

Figure 14 shows Mandel's Q-factor described by equation (39) versus $\varepsilon$ and $\eta$ for $A=100, \kappa=0.8$, and $\bar{n}_{t h}=0.15$. This figure indicates that Mandel's $Q$-factor for the given system is definitely positive. This implies that the generated laser light demonstrates super-Poissonian photon statistics. From this plot, we see that Mandel's Q-factor increased with the parametric amplifier at the minimum atomic coherence $\eta$, which is the maximum squeezing occurring point.

The result presented in Figure 15 indicates that Mandel's $Q$-factor increases with the decreasing value of the atomic coherence $\eta$ and with increasing the mean photon number of the thermal light $\bar{n}_{t h}$. However, Figure 16 shows a plot of Mandel's Q-factor of equation (39) versus $\varepsilon$ and $A$ for $\eta=0.1, \kappa=0.8$, and $\bar{n}_{t h}=0.15$. The result in this figure indicates that the linear gain coefficient (rate of atomic injection) increases the values of Mandel's $Q$-factor with at the maximum atomic coherence $\eta$. In these two figures, we observe that the value of Mandel's Q-factor is positive.

Moreover, as it can be described in Figure 17, the value of Mandel's Q-factor enhanced with the amplitude of the 


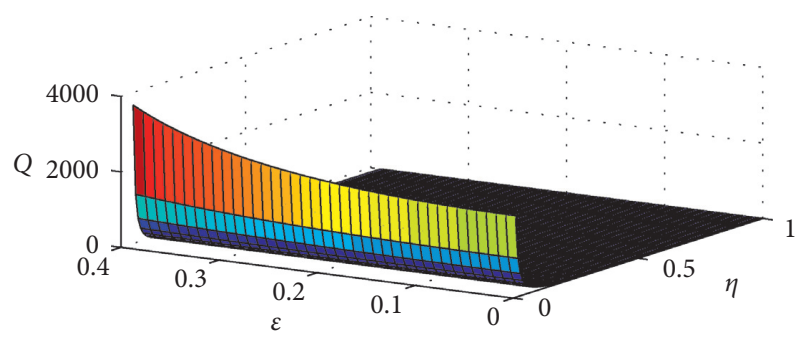

Figure 14: A plot of Mandel's $Q$-factor (equation (39)) versus $\varepsilon$ and $\eta$ for $A=100, \kappa=0.8$, and $\bar{n}_{t h}=0.15$.

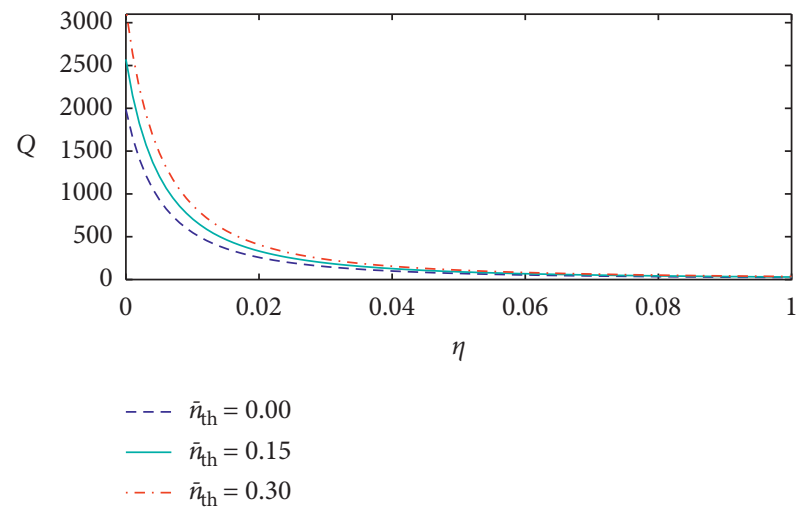

FIGURE 15: Plots of Mandel's $Q$-factor (equation (39)) versus $\eta$ for $A=100, \kappa=0.8, \varepsilon=0.399$ and for different values of $\bar{n}_{t h}$.

coherent light $\varepsilon$. Therefore, in these two figures, we observe that both the thermal reservoir and the driving coherent light increase the value of Mandel's Q-factor.

5.3. Photon Number Correlations. The normalized secondorder correlation function for the two-mode light can be expressed as [39]

$$
g_{\left(a_{1}, a_{2}\right)}^{(2)}(0)=\frac{\left\langle\widehat{a}_{1}^{\dagger} \widehat{a}_{2}^{\dagger} \widehat{a}_{1} \widehat{a}_{2}\right\rangle}{\left\langle\widehat{a}_{1}^{\dagger} \widehat{a}_{1}\right\rangle\left\langle\hat{a}_{2}^{\dagger} \hat{a}_{2}\right\rangle} .
$$

We realize that the operators in (39) are in the normal order. Therefore, the second-order correlation function can be expressed in terms of the $c$-number variables associated with the normal ordering as

$$
g_{\left(a_{1}, a\right)_{2}}^{(2)}(0)=1+\frac{\left\langle\alpha_{1}(t) \alpha_{2}(t)\right\rangle^{2}}{\left\langle\alpha_{1}^{*}(t) \alpha_{1}(t)\right\rangle\left\langle\alpha_{2}^{*}(t) \alpha_{2}(t)\right\rangle} .
$$

We plot, in Figure 18, the second-order correlation function of the two-mode light versus $\eta$ for $A=100, \kappa=0.8$, and $\varepsilon=0.399$ in the presence and absence of the thermal light $\bar{n}_{t h}$. We easily see from this figure that $g_{\left(a_{1}, a\right)_{2}}^{(2)}(0)$ increases with $\eta$ in both cases.

On the other hand, the plots in Figure 19 indicate that the parametric oscillator decreases the second-order correlation function for the same value of $\eta$. As this function deals with the correlation between the photon numbers, as already seen in the plots, it would not be a direct measure of the squeezing as well as entanglement of the given system.

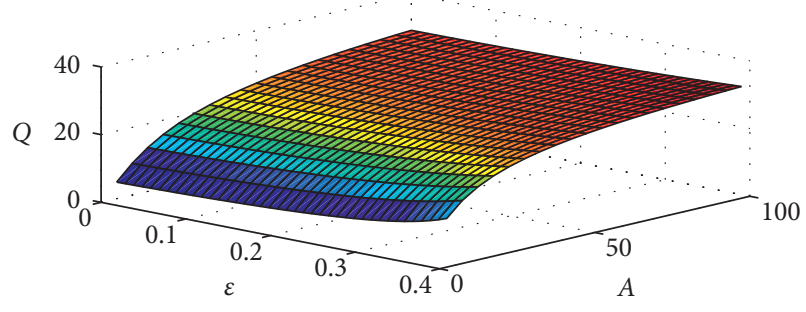

Figure 16: A plot of Mandel's $Q$-factor (equation (39)) versus $\varepsilon$ and $A$ for $\eta=0.1, \kappa=0.8$, and $\bar{n}_{t h}=0.15$.

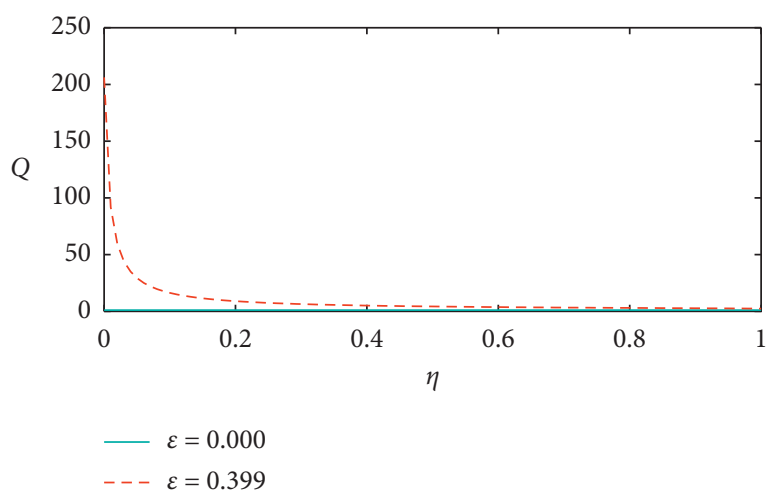

Figure 17: Plots of Mandel's $Q$-factor (equation (39)) versus $\eta$ for $A=10, \kappa=0.8, \bar{n}_{t h}=0.15$ and for different values of $\varepsilon$.

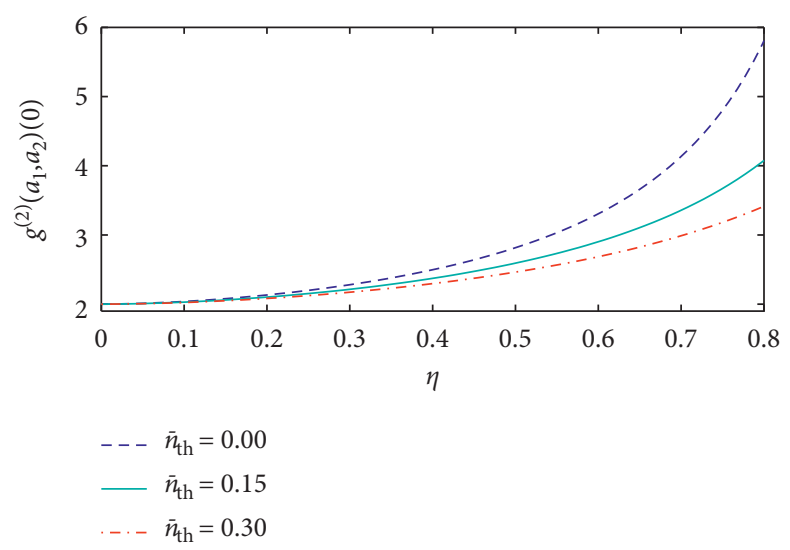

FIGURE 18: Plots of the normalized second-order correlation function (equation (41)) versus $\eta$ for $A=100, \kappa=0.8, \varepsilon=0.399$ and for different values of $\bar{n}_{t h}$.

Figure 20 shows the plots of the linear gain coefficient described by equation (41) versus $\eta$ and $A=100$ for $\kappa=0.8$, $\varepsilon=0.399$, and $\bar{n}_{t h}=0.15$. It is not difficult to see from this figure that the normalized second-order correlation function increases significantly with the linear gain coefficient and the atomic coherence $\eta$. From this, we conclude that the normalized second-order correlation of two-mode generated light increases as the degree of squeezing increases in general. Moreover, the presence of the parametric oscillator enhances the intermode correlation over the system. 


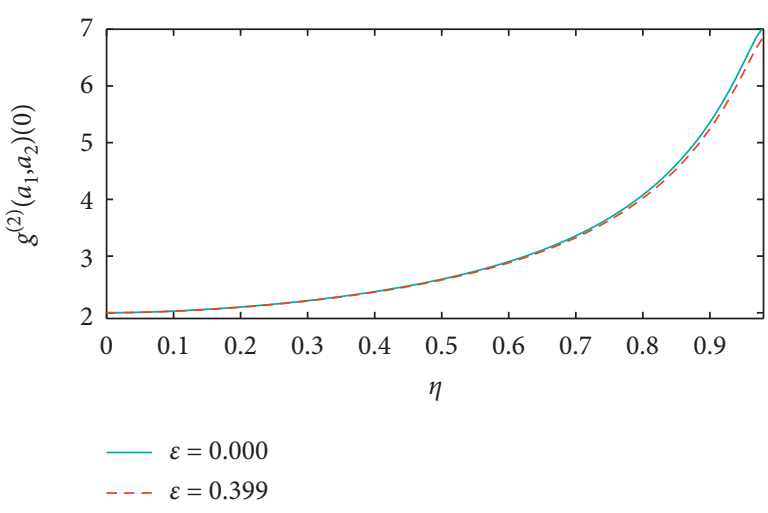

FIgURE 19: Plots of the normalized second-order correlation function (equation (41)) versus $\eta$ for $A=100, \kappa=0.8, \bar{n}_{t h}=0.15$ and for different values of $\varepsilon$.

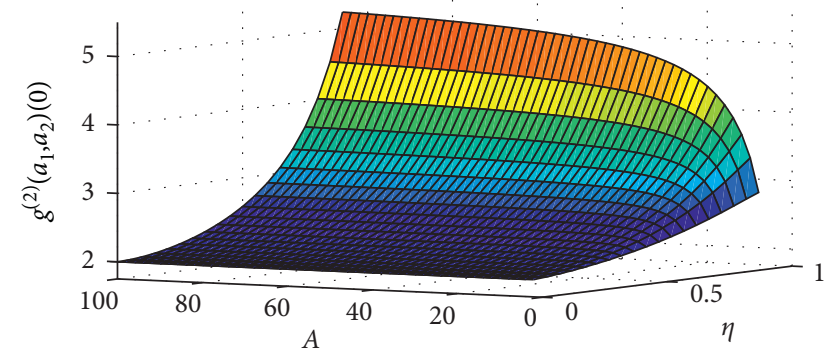

FIgURE 20: A plot of the normalized second-order correlation function (equation (41)) versus $\eta$ and $A=100$ for $\kappa=0.8$, $\varepsilon=0.399$, and $\bar{n}_{t h}=0.15$.

\section{Conclusion}

In this paper, we have studied the steady-state two-mode squeezing and entanglement of the light produced by a nondegenerate three-level cascade laser with a coherently driven parametric amplifier and coupled to a two-mode thermal reservoir in the linear and adiabatic approximation schemes in the good cavity limit. Here. we have considered a nondegenerate three-level laser in which the pump mode emerging from the parametric amplifier does not couple the top and bottom levels of the injected atoms [17]. This could be realized by putting on the right-side of the nonlinear crystal a screen which absorbs the pump mode. Moreover, we carry out our analysis by applying the master equation describing the dynamics of the optical system. Employing the solutions for $c$-number cavity mode variables along with the correlation property of noise forces associated with normal ordering, we obtained quadrature squeezing, the photon entanglement, the mean number of photon pairs, Mandel's Q-factor, and the second-order correlation functions of the two-cavity light.

It is found that the two-mode cavity radiation exhibits squeezing properties under certain conditions pertaining to the injected atomic coherence, where the degree of squeezing increases with the linear gain coefficient and the amplitude of the driving coherent light $\varepsilon$. However, the linear correlation coefficient increases with the degree of squeezing. But as the thermal light increases, the squeezing decreases. Moreover, we have found that the parametric amplifier produces a considerable degree of entangled light for a very small value of the linear gain coefficient regardless of how atoms are initially prepared. Generally, we have found that the degree of entanglement for the two-mode cavity light is directly related to the two-mode squeezing. Whenever there is squeezing in the two-mode light, there exists entanglement in the system. Since the parametric amplifier introduces additional squeezing to the system, the degree of entanglement has been enhanced for the given system. We have showed that the normalized second-order correlation of two-mode generated light increases as the degree of squeezing increases in general. Moreover, the presence of the parametric oscillator enhances the correlation of the two cavity light modes over the system. Finally, the quantum discords as a quantum correlation property can be investigated in the future.

\section{Data Availability}

The data used to support the findings of this study are included within the manuscript.

\section{Conflicts of Interest}

The authors declare that there are no conflicts of interest regarding the publication of this paper.

\section{Acknowledgments}

The authors thank Jimma University, College of Natural Sciences Research and Postgraduate Coordination Office, for their help, encouragement, and financial support during the research. This research was funded by Jimma University, College of Natural Sciences Research and Postgraduate Coordination Office.

\section{References}

[1] C. W. Gardiner, "Inhibition of atomic phase decays by squeezed light: a direct effect of squeezing," Physical Review Letters, vol. 56, no. 18, pp. 1917-1920, 1986.

[2] S. Qamar, S. Qamar, and M. S. Zubairy, "Effect of phase fluctuations on entanglement generation in a correlated emission laser with injected coherence," Optics Communications, vol. 283, no. 5, pp. 781-785, 2010.

[3] E. Alebachew, "Enhanced squeezing and entanglement in a non-degenerate three-level cascade laser with injected squeezed light," Optics Communications, vol. 280, no. 1, pp. 133-141, 2007.

[4] S. Tesfa, "Effect of dephasing on quantum features of the cavity radiation of an externally pumped correlated emission laser," Physical Review A, vol. 79, no. 6, Article ID 063815, 2009.

[5] N. A. Ansari, J. Gea-Banacloche, and M. S. Zubairy, "Phasesensitive amplification in a three-level atomic system," Physical Review A, vol. 41, no. 9, pp. 5179-5186, 1990.

[6] H. Xiong, M. O. Scully, and M. S. Zubairy, "Correlated spontaneous emission laser as an entanglement amplifier," Physical Review Letters, vol. 94, pp. 023601-023604, 2005. 
[7] S. Qamar, M. Al-Amri, and M. S. Zubairy, "Entanglement in a bright light source via Raman-driven coherence," Physical Review A, vol. 79, no. 3, Article ID 013831, 2009.

[8] J. Anwar and M. S. Zubairy, "Quantum-statistical properties of noise in a phase-sensitive linear amplifier," Physical Review A, vol. 49, no. 1, pp. 481-484, 1994.

[9] C. A. Blockley and D. F. Walls, "Intensity fluctuations in a frequency down-conversion process with three-level atoms," Physical Review A, vol. 43, no. 9, pp. 5049-5056, 1991.

[10] N. Lu, F.-X. Zhao, and J. Bergou, "Nonlinear theory of a twophoton correlated-spontaneous-emission laser: a coherently pumped two-level-two-photon laser," Physical Review A, vol. 39, no. 10, pp. 5189-5208, 1989.

[11] B. Daniel and K. Fesseha, "The propagator formulation of the degenerate parametric oscillator," Optics Communications, vol. 151, no. 4-6, pp. 384-394, 1998.

[12] N. Geoffery, Introduction to Nonlinear Optics, Cambridge University Press, New York, NY, USA, 2011.

[13] B. Teklu, "Parametric oscillation with the cavity mode driven by coherent light and coupled to a squeezed vacuum reservoir," Optics Communications, vol. 261, no. 2, pp. 310-321, 2006.

[14] W. H. Louisell, Quantum Statistical Properties of Radiation, Wiley, New York, NY, USA, 1973.

[15] T. Abebe, "Enhancement of squeezing and entanglement in a non-degenerate three-level cascade laser with coherently driven cavity," Ukrainian Journal of Physics, vol. 63, no. 8, p. 733, 2018.

[16] F. Kassahun, Fundamental of Quantum Optics, Dover Publications Inc., Austin, TX, USA, 2008.

[17] E. Alebachew, "Continuous-variable entanglement in a nondegenerate three-level laser with a parametric oscillator," Physical Review A, vol. 76, no. 2, Article ID 023808, 2007.

[18] T. Abebe, "The quantum analysis of nondegenerate three-level laser with spontaneous emission and noiseless vacuum reservoir," Ukrainian Journal of Physics, vol. 63, no. 11, pp. 969-978, 2018.

[19] T. Abebe, "Coherently driven nondegenerate three-level laser with noiseless vacuum reservoir," Bulgarian Journal of Physics, vol. 45, no. 4, pp. 357-373, 2018.

[20] S. Tesfa, "Effects of decoherence on entanglement in a correlated emission laser," Journal of Physics B: Atomic, Molecular and Optical Physics, vol. 40, no. 12, pp. 2373-2384, 2007.

[21] L.-M. Duan, G. Giedke, J. I. Cirac, and P. Zoller, "Inseparability criterion for continuous variable systems," Physical Review Letters, vol. 84, no. 12, pp. 2722-2725, 2000.

[22] S. Tesfa, "Dynamics of the cavity radiation of a correlated emission laser initially seeded with a thermal light," Physica Scripta, vol. 84, no. 4, Article ID 045403, 2011.

[23] L. Calderin, M. A. Hasan, N. G. Jenkins et al., "Experimental demonstration of coherent superpositions in an ultrasonic pseudospin," Scientific Reports, vol. 9, no. 1, 2019.

[24] S. Tesfa, "Entanglement amplification in a nondegenerate three-level cascade laser," Physical Review A, vol. 74, no. 4, Article ID 043816, 2006.

[25] E. A. Sete, "Bright entangled light from two-mode cascade laser," Optics Communications, vol. 281, no. 24, pp. 61246129, 2008.

[26] M. Hillery and M. S. Zubairy, "Entanglement conditions for two-mode states," Physical Review Letters, vol. 96, no. 5, Article ID 050503, 2006.

[27] J. Fiurasek and N. J. Cerf, "How to measure squeezing and entanglement of Gaussian states without homodyning," Physical Review Letters, vol. 93, no. 6, Article ID 063601, 2004.
[28] A. Serafini, F. Illuminati, and S. de Siena, "Symplectic invariants, entropic measures and correlations of Gaussian states," Journal of Physics B: Atomic, Molecular and Optical Physics, vol. 37, no. 2, pp. 21-28, 2004.

[29] J. Korbicz, C. I. Cirac, and M. Lewenstein, "Spin squeezing inequalities and entanglement of $N$ Qubit states," Physical Review Letters, vol. 95, no. 25, Article ID 120502, 2005.

[30] M. Horodecki, P. Horodecki, and R. Horodecki, "Separability of mixed states: necessary and sufficient conditions," Physics Letters A, vol. 223, no. 1-2, pp. 1-8, 1996.

[31] A. Peres, "Separability criterion for density matrices," Physical Review Letters, vol. 77, no. 8, pp. 1413-1415, 1996.

[32] R. Simon, "Peres-horodecki separability criterion for continuous variable systems," Physical Review Letters, vol. 84, no. 12 , pp. 2726-2729, 2000.

[33] S. Mancini, V. Giovannetti, D. Vitali, and T. Tombesi, "Entangling macroscopic oscillators exploiting radiation pressure," Physical Review Letters, vol. 88, no. 12, Article ID 120401, 2002.

[34] M. G. Raymer, A. C. Funk, B. C. Sanders, and H. de Guise, "Separability criterion for separate quantum systems," Physical Review A, vol. 67, no. 5, Article ID 052104, 2003.

[35] V. Giovannetti, S. Mancini, D. Vitali, and P. Tombesi, "Characterizing the entanglement of bipartite quantum systems," Physical Review A, vol. 67, no. 2, Article ID 022320, 2003.

[36] E. Shchukin and W. Vogel, "Inseparability criteria for continuous bipartite quantum states," Physical Review Letters, vol. 95, no. 24, Article ID 230502, 2005.

[37] G. Adesso, A. Serafini, and F. Illuminati, "Extremal entanglement and mixedness in continuous variable systems," Physical Review A, vol. 70, no. 2, Article ID 022318, 2004.

[38] G. Vidal and R. F. Wener, "Computable measure of entanglement," Physical Review A, vol. 65, no. 3, Article ID 032314, 2002.

[39] M. Fox, Quantum Optics: An Introduction, Oxford University Press, Oxford, UK, 2006. 